\title{
Modeling the inactivation of Bacillus subtilis spores by ethylene oxide processing
}

\author{
G. C. Mendes • T. R. S. Brandão • C. L. M. Silva
}

\begin{abstract}
Ethylene oxide is currently a dominant agent in medical device sterilization. This work intends to study the main effects and interactions of temperature, ethylene oxide concentration, and relative humidity on commercial spore strips of Bacillus subtilis, var. niger (ATCC 9372) inactivation, the most common microorganism used in controlling the efficacy of the process. Experiments were carried out using a full factorial experimental design at two levels $\left(2^{3}\right.$ factorial design). Limit target exposure conditions for ethylene oxide concentration, temperature, and relative humidity were $250-1,000 \mathrm{mg} \mathrm{EO} / \mathrm{l}, 40-60^{\circ} \mathrm{C}$, and $50-90 \%$, respectively. Adopting a different approach from the first-order kinetics, a Gompertz model was successfully applied in data fitting of the inactivation curves. Bacillus subtilis kinetic behavior presented a sigmoidal inactivation with an initial shoulder $(\lambda)$, followed by a maximum inactivation rate $\left(\mathrm{k}_{\max }\right)$, these being model parameters. It was concluded that temperature and ethylene oxide concentration were the most significant factors and consequently, additional experiments were carried out aiming at describing the parameters' dependence on these process factors. Mathematical relations describing such dependences were successfully developed and included in the Gompertz kinetic model. The predictive ability of this
\end{abstract}

\author{
G. C. Mendes \\ Bastos Viegas, S.A., Avenida da Fábrica 298, \\ Guilhufe, 4564-155 Penafiel, Portugal \\ G. C. Mendes · T. R. S. Brandão · C. L. M. Silva ( () \\ Centro de Biotecnologia e Química Fina, \\ Escola Superior de Biotecnologia, \\ Universidade Católica Portuguesa, \\ Rua Dr. António Bernardino de Almeida, \\ 4200-072 Porto, Portugal \\ e-mail: clsilva@esb.ucp.pt
}

integrated model was assessed, and its adequacy in predicting B. subtilis inactivation was proven.

Keywords Applied microbiology · Modeling ·

Bacillus subtilis spores · Ethylene oxide sterilization

\section{Introduction}

Sterility is a critical attribute of several medical devices [31]. Nowadays, ethylene oxide (EO) is a dominant sterilization agent used in the medical-device industry and its volume is increasing due to the growth of single-use MDs and especially because there has been a transition to customized packs for use in specific medical and surgical procedures.

The big advantages of this sterilization process is its effectiveness and compatibility with most materials, together with its flexibility, resulting from its dependency on several factors, such as concentration, temperature (T), humidity, and time (and their combinations) [20]. Understanding the full dynamics of the sterilization allows the definition of appropriate process variables, thus contributing to its design optimization.

Bacillus subtilis, due to its resistance to EO sterilization, is the most common microorganism used in the process control [14]. Heider et al. [13] described a linear relation between the inactivation rate and EO concentration (in the range $50-1,200 \mathrm{mg} / \mathrm{l}$ ) and a similar tendency was observed for temperature (in the range $30-64^{\circ} \mathrm{C}$ ). The temperature and $\mathrm{EO}$ concentration were independent variables.

Relative humidity $(\mathrm{RH})$ is a complex process variable and contradictory results were obtained by different authors [24, 27, 31]. Recent findings show that within the limits of $30-90 \%$, the RH does not influence lethality 
$[13,24,26,31]$. Out of these limits, at very humid or dry environments, the diffusivity of EO into devices and microbes is compromised [24-26].

The microbial inactivation by EO sterilization has been considered to follow a first-order kinetics, although model prediction was scarcely (or never) assessed. According to the first-order kinetics approach, a plot of the logarithmic of the number of survival microorganisms versus time is a straight line. The reciprocal of the slope is the well-known D-value (i.e., the required time for a tenfold of the microbial load). However, a number of studies report deviations to the linear behavior [16, 28]. To circumvent these situations, some authors excluded the initial shoulder period of the experimental data $[17,33]$ in data fitting, while others forget the convenient regression analysis procedures by performing linear regression to obvious nonlinear experimental data patterns. Therefore, extrapolations of predicted times based on reported D-values from linear models may not be appropriate.

Deviations from linearity can be assumed as a complete (or incomplete) sigmoidal behavior with the following features: shoulder time (or lag) period, prior to a linear phase corresponding to a maximum growth/inactivation rate, followed by a tail (asymptotic phase). A number of models have been used to describe these sigmoidal tendencies [5, 34]: Weibull and logistic functions [4, 7, 29], Gompertz equation [2], and Baranyi and Geeraerd models [1, 10].

Gompertz function, and its modified forms, has the ability of modeling both linear and asymmetrical sigmoidal data. For the inactivation behavior, the following Gompertz function $[2,6,9,11,12,18,19]$ can be used:

$\log \left(\frac{\mathrm{N}}{\mathrm{N}_{0}}\right)=\mathrm{A} \cdot \exp \left[-\exp \left\{\frac{-\mathrm{k}_{\max } \mathrm{e}}{\mathrm{A}}(\lambda-\mathrm{t})+1\right\}\right]$

where $\mathrm{N}$ is the microbial load at a particular process time $\mathrm{t}$ (the index 0 is related to initial microbial load). The model parameters are the maximum inactivation rate, $\mathrm{k}_{\max }$, and shoulder period, $\lambda$. The A value is the asymptotic response, being considered the tail (i.e., a resistant residual microbial population). It is not consensual whether the tail is actually a residual population or an enumeration method limitation. The Gompertz model is sometimes referred to as being inadequate in predicting initial microbial load (i.e., for $\mathrm{t}=0, \log \left(\mathrm{N} / \mathrm{N}_{0}\right)$ only approaches zero $)$. However, the overor sub-estimation of this value may be negligible when compared to the experimental variations related to enumeration methods.

Studies on the influence of the process variables on microbial inactivation kinetics using ethylene oxide are lacking. Quantification of the kinetic parameters is obtained, as well as effect of relevant factors on their estimates. Consequently, the objectives of the present study were to: (1) screen the most significant variables on
B. subtilis inactivation by EO sterilization, (2) model the inactivation kinetics of B. subtilis including the variables' effects and, (3) provide a method of integrating lethality, thus contributing to the design optimization and efficient control of the inactivation processes. This is certainly important when moving towards parametric release, i.e., the approval of the process relying merely on the measurement and assessment of process variables [15].

\section{Materials and methods}

Experimental procedures

\section{Experimental design}

Experiments were carried out in an EO sterilizer (please see Sterilization process section) and conditions were defined according to a full factorial experimental design at two levels of three factors ( $2^{3}$ factorial design) [3]. The parameters used for each condition, including the time of each step, with the exception of the gas exposure time, were kept constant. Three independent variables representing temperature, EO concentration, and humidity were assumed, each variable tested in two levels: a high level (+) and a low level (-), according to Table 1 (totaling eight experimental conditions, corresponding to runs 1-8). Target limits for the exposure conditions of $\mathrm{EO}$ concentration, temperature, and relative humidity were $250-1,000 \mathrm{mg} \mathrm{EO} / 1,40-60^{\circ} \mathrm{C}$, and $50-90 \%$, respectively. The limits chosen for the process variables were based on literature review [13, 24, 32, 34] and operating conditions of the sterilizers. However, difficulties arise in stabilizing the process conditions and actual attained operating values are in Table 1.

The analysis of variance (ANOVA) allowed identifying the most significant parameters affecting microbial inactivation during EO sterilization and additional experiments considering intermediate conditions of these parameters were defined in order to model their effects and combined effects on the lethality (runs 9-15 included in Table 1).

\section{Bacterial strain}

Spore strip biological indicators (SGMStrip $^{\mathrm{TM}}$, SGM Biotech, Inc., Montana, USA) were used in inactivation processes by EO. The biological indicators (BI) contain a known number of Bacillus subtilis, var. niger (ATCC 9372) spores (magnitude of $10^{6}$ colony-forming units (CFU), corresponding to an initial unprocessed microbial load, $\mathrm{N}_{0}$ ) inoculated onto filter paper. Compliance with USP, ISO 11138, and EN 866 was verified.

The BI recovery technique was validated to obtain reliable spores enumeration. The recovery of spores from 
Table 1 Estimated $\mathrm{k}_{\max }$ and $\lambda$ parameters of $B$. subtilis inactivation at the temperature, EO concentration, and relative humidity conditions tested

\begin{tabular}{|c|c|c|c|c|c|c|c|c|c|c|c|}
\hline \multirow{3}{*}{$\begin{array}{l}\text { Run } \\
1\end{array}$} & \multicolumn{6}{|c|}{ Variables } & \multicolumn{4}{|l|}{ Parameters } & \multirow{3}{*}{$\begin{array}{l}\begin{array}{l}\text { Regression } \\
\text { analysis R }\end{array} \\
0.992\end{array}$} \\
\hline & \multicolumn{2}{|c|}{$\mathrm{T}\left({ }^{\circ} \mathrm{C}\right)$} & \multicolumn{2}{|c|}{ [EO] (mg/l) } & \multicolumn{2}{|c|}{$\mathrm{RH}(\%)$} & \multirow{2}{*}{$\frac{\mathrm{k}_{\max } \times 10^{3}\left(\mathrm{~s}^{-1}\right)}{4.56}$} & \multirow{2}{*}{$\frac{\mathrm{SHW}_{95 \%}}{3.01}$} & \multirow{2}{*}{$\frac{\lambda(\mathrm{s})}{391}$} & \multirow{2}{*}{$\frac{\text { SHW }_{95 \%}}{5}$} & \\
\hline & 60 & $(+)$ & 233 & $(-)$ & 63 & $(-)$ & & & & & \\
\hline 2 & 44 & $(-)$ & 257 & $(-)$ & 86 & $(+)$ & 1.78 & 2.42 & 1,079 & 3 & 0.993 \\
\hline 3 & 34 & $(-)$ & 222 & $(-)$ & 60 & $(-)$ & 0.989 & 2.93 & 1,179 & 7 & 0.988 \\
\hline 4 & 40 & $(-)$ & 980 & $(+)$ & 90 & $(+)$ & 3.49 & 2.66 & 418 & 5 & 0.991 \\
\hline 5 & 59 & $(+)$ & 266 & $(-)$ & 83 & $(+)$ & 4.46 & 3.56 & 353 & 7 & 0.991 \\
\hline 6 & 33 & $(-)$ & 940 & $(+)$ & 61 & $(-)$ & 2.16 & 2.50 & 605 & 6 & 0.989 \\
\hline 7 & 59 & $(+)$ & 1004 & $(+)$ & 98 & $(+)$ & 7.65 & 8.59 & 266 & 17 & 0.983 \\
\hline 8 & 60 & $(+)$ & 977 & $(+)$ & 46 & $(-)$ & 10.00 & $*$ & 0 & $*$ & $*$ \\
\hline 9 & 37 & & 674 & & 73 & & 2.28 & 2.72 & 831 & 4 & 0.991 \\
\hline 10 & 37 & & 456 & & 80 & & 1.83 & 2.57 & 821 & 5 & 0.991 \\
\hline 11 & 51 & & 247 & & 80 & & 3.23 & 3.60 & 482 & 7 & 0.985 \\
\hline 12 & 51 & & 447 & & 67 & & 4.09 & 3.37 & 300 & 9 & 0.994 \\
\hline 13 & 50 & & 675 & & 72 & & 5.04 & 4.94 & 257 & 15 & 0.992 \\
\hline 14 & 60 & & 738 & & 71 & & 7.33 & 8.01 & 255 & 18 & 0.994 \\
\hline 15 & 62 & & 498 & & 77 & & 5.89 & 6.25 & 291 & 12 & 0.988 \\
\hline
\end{tabular}

* Meaningless value

control samples (not submitted to the sterilization process) was $70 \%$ of the analysis report of the BI manufacturer.

\section{Sterilization process}

All experiments were performed in a standard EO chamber (21059C Sterichem, France) of approximately $3 \mathrm{~m}^{3}$ with controlled temperature, EO concentration, and humidity. These conditions were maintained homogeneous inside the chamber due to forced recirculation, and were monitored by adequate equipment.

Temperature and relative humidity were monitored inside the load, using Kaye ValProbe ${ }^{\circledR}$ wireless data loggers, part numbers XVP-L-T and X2520, respectively. EO concentration was assessed by an infrared analyzer in the sterilizer chamber and corresponds to a condition obtained in the sterilizer headspace, since the techniques currently available to the industry cannot measure this parameter inside the load, where the lethality of the process is being monitored.

For each of the defined exposure conditions, the sterilization cycle was performed under vacuum, using a mixture of EO (Avantec, France) and nitrogen (Air Liquide, Portugal) with a minimum purity of 99.9 and $99.5 \%$, respectively. EO was the sterilization agent and nitrogen was applied to create a neutral atmosphere, avoiding flammable atmosphere inside the sterilization chamber.

The sporicidal activity of a specific EO sterilization cycle was assessed by placing the inoculated paper carriers with about $10^{6} \mathrm{~B}$. subtilis spores into the middle of peelpacks of drape material (488-103, Bastos Viegas S. A,
Portugal) adjacent to temperature and humidity sensors. BI samples were removed after different exposure times to the sterilizing agent. Enumeration of the viable spores was performed according to the procedure described bellow.

\section{Enumeration of viable spores}

Enumeration of bacterial viable spores was performed in a laminar air flow cabinet (AH-100 Telstar $^{\circledR}$, Spain). Exposed spore carriers were transferred to a sterile screw cap, flat bottom tube with six glass beds of $5 \mathrm{~mm}$ containing $10.0 \mathrm{ml}$ of sterile purified water (ten dilution) thus, the minimal detection limit is $10 \mathrm{CFU} / \mathrm{ml}$. Tubes were vortexed for approximately $4-5 \mathrm{~min}$ (until the paper carrier was macerated to pulp) followed by a heat shock. Tubes were placed in a thermostatic bath (at $80-85^{\circ} \mathrm{C}$ ) for $10 \mathrm{~min}$ and rapidly transferred into an ice bath $\left(\right.$ at $\left.0-4^{\circ} \mathrm{C}\right)$.

Samples were serially diluted and plated in triplicate onto tryptone soya agar (OXOID, UK). All plates were incubated at $32 \pm 2^{\circ} \mathrm{C}$ and counted after $72 \mathrm{~h}$ (microbial load $\left.\mathrm{N}\right)$.

The inactivation data was normalized in relation to initial load (please see Bacterial strain section) and expressed as $\operatorname{logarithms~(i.e.,~} \log \left(\mathrm{N} / \mathrm{N}_{0}\right)$ ).

\section{Modeling procedures}

\section{Equivalent time}

Since standard-sized process chambers do not produce square wave cycles, and significant lethality may occur 
throughout gas injection and exhaust phases, an equivalent exposure time, $\mathrm{U}$, rather than exposure time, $t$, should be considered (in Eq. 1). The following expression can be used for its estimation [21, 22, 24, 26]:

$\mathrm{U}=\frac{\mathrm{t}_{\text {injection }}}{2}+\mathrm{t}_{\text {nitrogen overlay }}+\mathrm{t}+\frac{\mathrm{t}_{\text {exhaust }}}{2}$

assuming a constant rate of pressure increase (in the injection phase) and pressure decrease (in the exhaust phase).

Some authors $[22,23,26]$ refer that the initial shoulder observed in the inactivation data is related to the consideration of the exposure time rather than the equivalent time.

Regression analysis and statistical assessment

The Gompertz model (Eq. 1) was used to fit experimental inactivation data of $\log \left(\mathrm{N} / \mathrm{N}_{0}\right)$. The non-linear regression analysis was performed using the Levenberg-Marquardt algorithm to minimize the sum of the squares of the differences between the predicted and experimental values. Model parameters (i.e., $\mathrm{k}_{\max }$ and $\lambda$ ) were estimated and their precision was evaluated by confident intervals by the standardized half-width (SHW) at 95\%, i.e., halved confidence interval divided by the estimate $\left(\mathrm{SHW}_{95 \%} \equiv\right.$ $\frac{\text { confidence interval }_{95 \%}}{2} \times \frac{1}{\text { estimate }} \times 100$ ).

The quality of the regression was assessed by residuals analyses (normality and randomness) and by the coefficient of determination $\mathrm{R}^{2}$.

Results from $2^{3}$ factorial experimental design were analyzed by ANOVA procedures.

Statistica ${ }^{\odot} 6.0$ (StatSoft, USA), and Microsoft ${ }^{\circledR}$ Excel 2000 (Microsoft Corporation, USA) were used for all calculations and statistical analysis.

\section{Results and discussion}

Influence of environmental conditions on microbial inactivation kinetics

The influence of EO concentration, temperature, and relative humidity on inactivation behavior of $B$. subtilis spores was studied in a preliminary step using the conditions defined according to the $2^{3}$ experimental design (Table 1 ; Fig. 1). The shape of the inactivation curves depends on the lethal agent intensity but, in general, significant deviations from linearity are evident and the general shape is a concave downward curve. As observed, the data exhibit an initial shoulder prior to exponential phase of death. Forcing a straight line through the experimental non-linear survival curves is obviously an undesirable option, and can lead to a considerable error of underestimation of process time when combined with extrapolation. The derived estimated times needed to obtain the target lethality seem to be higher than predictions derived from D-values calculations on "linear" inactivation curves and this should be avoided.

A Gompertz model was chosen due to its versatility in describing different tendencies (from linear to pronounced sigmoidal shapes), depending on the magnitude of the model parameters. Results showed that experimental inactivation data were successfully fitted with the Gompertz model and estimated parameters (i.e., $\lambda$ and $\mathrm{k}_{\max }$ ) are included in Table 1 . The goodness of model fitting was assessed on the basis of residuals randomness and normality (which was verified in all cases) and on the coefficient of determination $\left(\mathrm{R}^{2}\right.$ was greater than 0.98 , meaning that at least $98 \%$ of the observed variability was explained by the model).

The overall inactivation curves were divided into a slower first stage (i.e., a shoulder phase) and in a second stage for exponential inactivation (i.e., maximum death rate). A true tailing was not observed under the conditions tested. The residual final value could be defined due to an enumeration method limitation and does not correspond to a residual resistant population. Consequently, the tail was not defined as a model parameter, and an asymptotic value of -7.5 was assumed (reflects overall tendency in all experiments and avoids interference with the studied kinetic parameters).

High precision of $\mathrm{k}_{\max }$ and $\lambda$ estimates was attained (evaluated by $\mathrm{SHW}_{95 \%}$ ). The poor results were obtained for conditions of run 7 with a standard half-width of $8.6 \%$ for $\mathrm{k}_{\max }$ (the remaining values averaging $2.8 \%$ ) and $16.8 \%$ for $\lambda$ (the remaining values averaging 5.7\%). For both, $\lambda$ and $\mathrm{k}_{\max }$, higher errors tend to be obtained for the highest temperatures and EO concentration. This is reasonable since, for a higher lethal condition, the inactivation is faster and, consequently, there is a lack of experimental points, mainly in the initial period, which implies lower precision in the estimation of parameters.

The factorial experimental design allowed concluding about the process variables (and combination of them) that significantly affected the inactivation kinetics of $B$. subtilis behavior (assessed by $\mathrm{k}_{\max }$ and $\lambda$ parameters). Results showed that temperature had the most significant effect on $\mathrm{k}_{\max }$ and $\lambda$, followed by EO concentration (at a significance level of 15\%). Therefore, special attention should be given to the accuracy of the temperature sensors used to monitor the sterilization conditions. The temperature and the EO concentration have a negative effect on $\lambda$ and a positive effect on $\mathrm{k}_{\max }$. This means that higher temperatures and EO concentration imply narrow shoulder times and higher inactivation rates. On the contrary, lower inactivation rates and more evident shoulder phases were observed at the 
Fig. 1 Inactivation of B. subtilis spores by EO sterilization at conditions defined according to the $2^{3}$ factorial design. Open circle experimental data; Thick solid line fitted Gompertz model (Eq. 1); Thin solid line predicted data by Eq. (5); Dotted line upper and lower limits of predicted data; a Run1: $\mathrm{T}=60^{\circ} \mathrm{C} ; \mathrm{EO}=233 \mathrm{mg} / \mathrm{l}$;

$\mathrm{RH}=63 \%$, b Run2: $\mathrm{T}=44^{\circ} \mathrm{C}$; $\mathrm{EO}=257 \mathrm{mg} / \mathrm{l} ; \mathrm{RH}=86 \%$, c Run3: $\mathrm{T}=34^{\circ} \mathrm{C}$;

$\mathrm{EO}=222 \mathrm{mg} / \mathrm{l} ; \mathrm{RH}=60 \%$, d Run4: $\mathrm{T}=40^{\circ} \mathrm{C}$;

$\mathrm{EO}=980 \mathrm{mg} / \mathrm{l} ; \mathrm{RH}=90 \%$, e Run5: $\mathrm{T}=59^{\circ} \mathrm{C}$;

$\mathrm{EO}=266 \mathrm{mg} / \mathrm{l} ; \mathrm{RH}=85 \%$,

f Run6: $\mathrm{T}=33^{\circ} \mathrm{C}$;

$\mathrm{EO}=940 \mathrm{mg} / \mathrm{l} ; \mathrm{RH}=61 \%$, g Run7: $\mathrm{T}=59^{\circ} \mathrm{C}$;

$\mathrm{EO}=1004 \mathrm{mg} / \mathrm{l} ; \mathrm{RH}=98 \%$
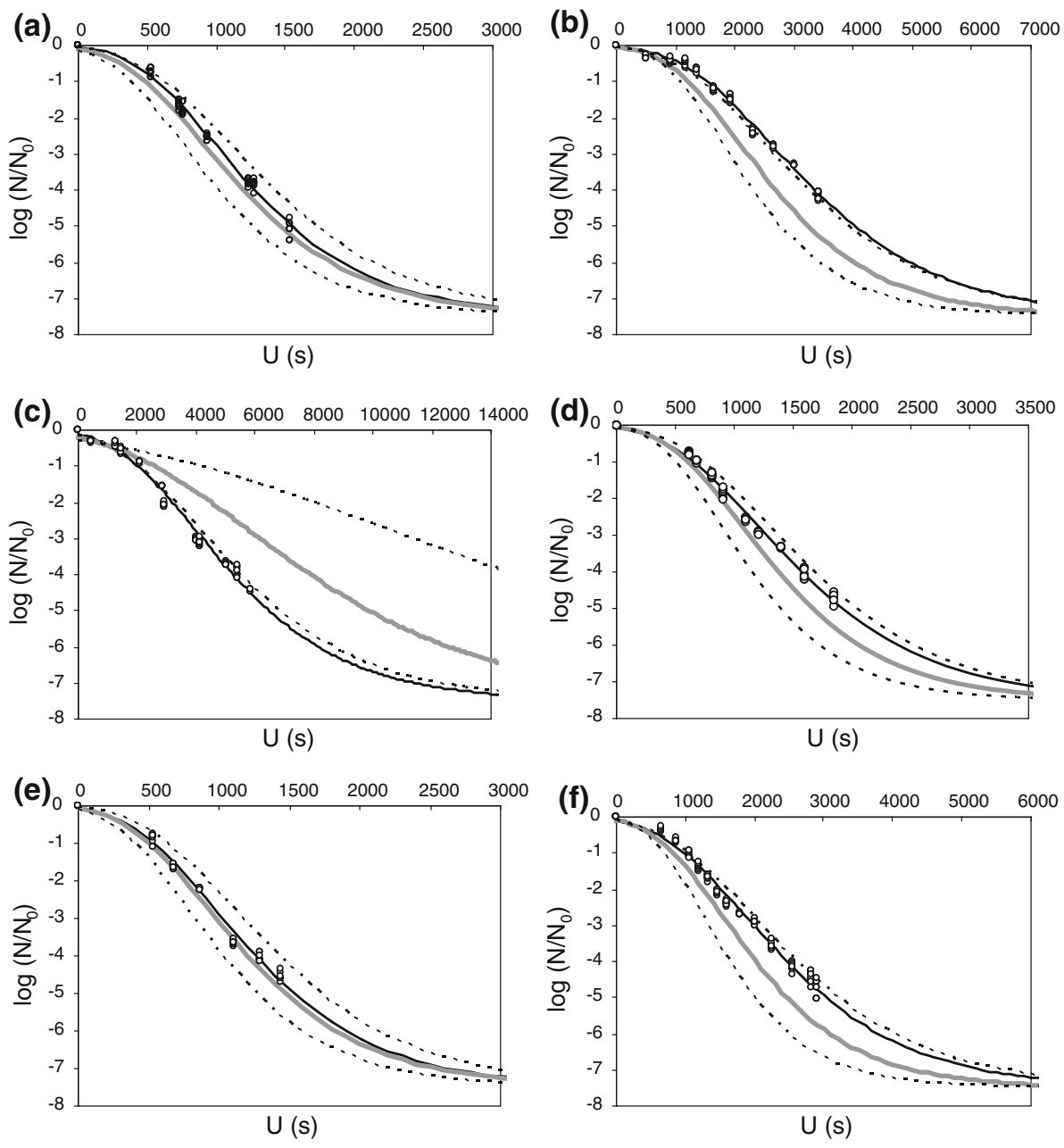

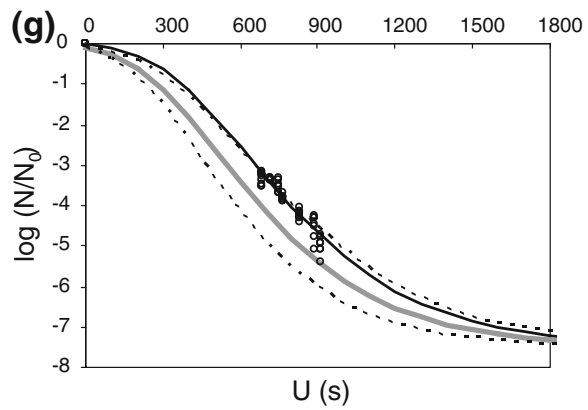

lowest temperature and EO concentration. Effects resulting from the combination of temperature and EO were not significant. Also, the relative humidity (and its combined effects with the remaining variables) did not influence significantly the inactivation of $B$. subtilis.

Based on these achievements, seven additional experimental conditions were tested using intermediate conditions of temperature and concentration, combined with the limits previously defined: one temperature (about $50^{\circ} \mathrm{C}$ ) and two more EO concentrations (about 470 and $700 \mathrm{mg} / \mathrm{l}$ ). These experiments provided important data aiming at describing more accurately the dependence of kinetic parameters on environmental conditions. These results are also included in Table 1 (runs 9-15) and inactivation curves in Fig. 2. Model adequacy and goodness of fits were assessed as previously mentioned. A good precision of parameter estimates was observed, since low errors were attained (maximum $\mathrm{SHW}_{95 \%}$ values of 8.0 and $17.8 \%$, respectively, for $\mathrm{k}_{\max }$ and $\lambda$, again for the highest variables values, with the remaining value averaging 3.9 and $8.5 \%$ for $\mathrm{k}_{\max }$ and $\lambda$, respectively).

Regarding temperature, $Q_{10}$ is the coefficient that defines the change in the reaction rate constant for a change in temperature of $10^{\circ} \mathrm{C}$. The reported $\mathrm{Q}_{10}$ for destroying 


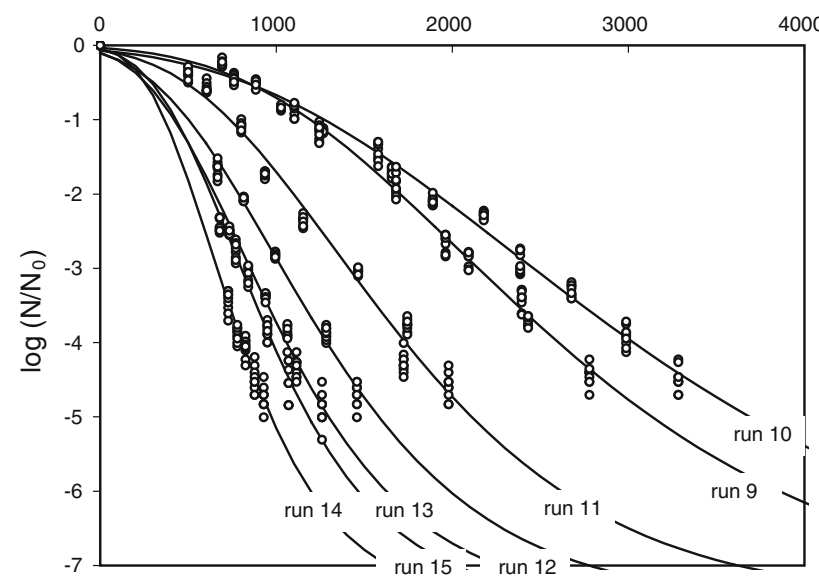

$U(\mathrm{~s})$

Fig. 2 Inactivation of B. subtilis spores by EO sterilization at the additional experimental conditions. Open circle experimental data; Solid line fitted Gompertz model (Eq. 5); Run9: $\mathrm{T}=37^{\circ} \mathrm{C}$; $\mathrm{EO}=674 \mathrm{mg} / \mathrm{l} ; \mathrm{RH}=73 \%$, Run10: $\mathrm{T}=37^{\circ} \mathrm{C} ; \mathrm{EO}=456 \mathrm{mg} / \mathrm{l} ;$ $\mathrm{RH}=80 \%$, Run $11: \mathrm{T}=51^{\circ} \mathrm{C} ; \mathrm{EO}=247 \mathrm{mg} / \mathrm{l} ; \mathrm{RH}=80 \%$, Run 12 : $\mathrm{T}=51{ }^{\circ} \mathrm{C} ; \quad \mathrm{EO}=447 \mathrm{mg} / \mathrm{l} ; \quad \mathrm{RH}=67 \%, \quad \operatorname{Run} 13: \quad \mathrm{T}=50^{\circ} \mathrm{C} ;$ $\mathrm{EO}=675 \mathrm{mg} / \mathrm{l} ; \mathrm{RH}=72 \%$, Run14: $\mathrm{T}=60^{\circ} \mathrm{C} ; \mathrm{EO}=738 \mathrm{mg} / \mathrm{l} ;$ $\mathrm{RH}=71 \%$, Run $15: \mathrm{T}=62^{\circ} \mathrm{C} ; \mathrm{EO}=498 \mathrm{mg} / \mathrm{l} ; \mathrm{RH}=77 \%$

spores with EO has been reported to be in the range of 1.4-2.0. A consensus seems to consider a value close to 2 for $\mathrm{Q}_{10}$, which means that a $10^{\circ} \mathrm{C}$ change would affect lethality by a factor of 2 and the $\mathrm{z}$-value would be very close to $30^{\circ} \mathrm{C}[8,13,24,25,30]$.

If one considers the pairs of runs with identical EO concentrations (i.e., runs $1-11,15-12$, and 14-13) and temperatures differing by $10^{\circ} \mathrm{C}$ (i.e., sterilization temperatures of 60 and $50^{\circ} \mathrm{C}$, respectively), the inactivation rate differs by a factor of 1.4. However, if other runs are compared (i.e., runs $2-3$ at 44 and $34^{\circ} \mathrm{C}$, respectively), the value obtained is 1.8 .

\section{Assessment of model prediction}

Temperature and EO concentration were the most significant factors affecting $B$. subtilis inactivation parameters. Consequently, equations that describe these influences on $\mathrm{k}_{\max }$ and $\lambda$ were developed aiming at obtaining a mathematical inactivation model expressed in terms of the relevant processing variables. It was assumed that $\mathrm{k}_{\max }$ varied linearly with EO concentration, for given temperatures $\left(\mathrm{k}_{\max }=\mathrm{a}_{\mathrm{k}}+\mathrm{b}_{\mathrm{k}}[\mathrm{EO}]\right)$. Some authors had reported a plateau effect in the inactivation rate within the range 250-1,000 mg/l, at higher ethylene oxide concentrations, but Mosley et al. [23, 25] did not observe such an effect. This last finding is in accordance with our results (Fig. 3a). Concerning the $\lambda$ parameter, the relation $\lambda=\mathrm{a}_{\lambda}+\mathrm{b}_{\lambda}$ $\ln [\mathrm{EO}]$ provided the best fits (Fig. 3b).

The parameters $a_{k}$ and $b_{k}$, as well as $a_{\lambda}$ and $b_{\lambda}$, were estimated by regression analysis procedures, and the influence of temperature on these estimates was studied. Linear relations of these parameters on temperature were defined (Fig. 4). The quality of the model fits were attained by residual analysis and $\mathrm{R}^{2}$ magnitude, which in all cases were above 0.90 .

Merging all of the developed equations, the final expressions that relate $\mathrm{k}_{\max }$ and $\lambda$ with temperature and $\mathrm{EO}$ concentration are as follows:

$$
\begin{aligned}
\mathrm{k}_{\max }= & \left(1.42 \times 10^{-4} \mathrm{~T}-4.96 \times 10^{-3}\right) \\
& +\left(5.54 \times 10^{-8} \mathrm{~T}+1.25 \times 10^{-6}\right)[\mathrm{EO}] \\
\lambda= & \left(1.63 \times 10^{1} \mathrm{~T}-1.06 \times 10^{3}\right) \ln ([\mathrm{EO}]) \\
+ & \left(-1.25 \times 10^{2} \mathrm{~T}+8.23 \times 10^{3}\right) .
\end{aligned}
$$

The predictive ability of these expressions were assessed by plotting the estimated values of $\mathrm{k}_{\max }$ and $\lambda$ based on experimental data fits using the Gompertz model (Eq. 1) versus the ones obtained using the previous equations (Fig. 5). It can be concluded that for $\mathrm{k}_{\max }$, Eq. 3 allows a good prediction (a strong agreement between $\mathrm{k}_{\max }$ estimated on the base of experimental data and the one expressed in terms of processing variables). Concerning $\lambda$, Eq. (4) allows a satisfactory prediction. A higher dispersion of $\lambda$ estimated on the base of experimental data and the one expressed in terms of processing variables was observed when compared to the results obtained for the inactivation rate. This is not unexpected, since the shoulder parameter is estimated with less precision (results previously discussed).

The final objective of this work was to express the inactivation data (i.e., $\log \left(\mathrm{N} / \mathrm{N}_{0}\right)$ ) in terms of the most significant processing variables (i.e., $\mathrm{T}$ and EO concentration). Consequently, Eqs. (3) and (4) were integrated in the Gompertz model (Eq. 1). The final expression was:

$$
\begin{aligned}
\log \left(\frac{\mathrm{N}}{\mathrm{N}_{0}}\right)= & (-7.5) \exp \left\{-\exp \left[\frac{-\left[\left(1.42 \times 10^{-4} \mathrm{~T}-4.96 \times 10^{-3}\right)+\left(5.54 \times 10^{-8} \mathrm{~T}+1.25 \times 10^{-6}\right)[\mathrm{EO}]\right] \mathrm{e}}{-7.5}\right.\right. \\
& \left.\left.\times\left[\left(1.63 \times 10^{1} \mathrm{~T}-1.06 \times 10^{3}\right) \ln ([\mathrm{EO}])+\left(-1.25 \times 10^{2} \mathrm{~T}+8.23 \times 10^{3}\right)-\mathrm{U}\right]+1\right]\right\}
\end{aligned}
$$


Fig. 3 Influence of EO concentration on kinetic parameters, $\mathrm{k}_{\max }$, and $\lambda$. a Influence of EO concentration on $\mathrm{k}_{\max }$. b Influence of EO concentration on $\lambda$
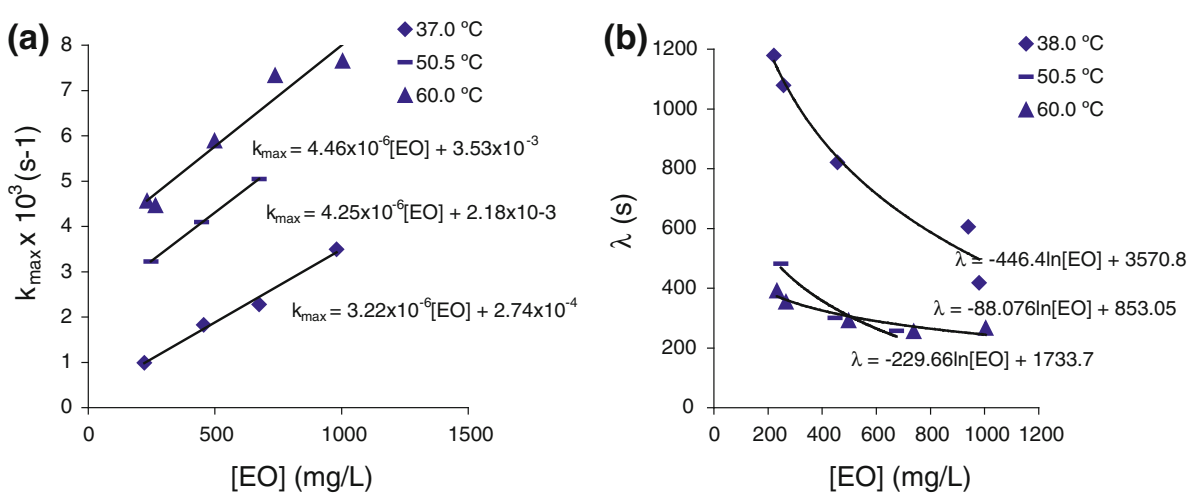

Fig. 4 Influence of $T$ on $a_{k}$ and $\mathrm{b}_{\mathrm{k}}(\mathbf{a})$ and $\mathrm{a}_{\lambda}$ and $\mathrm{b}_{\lambda}$

(b) parameters
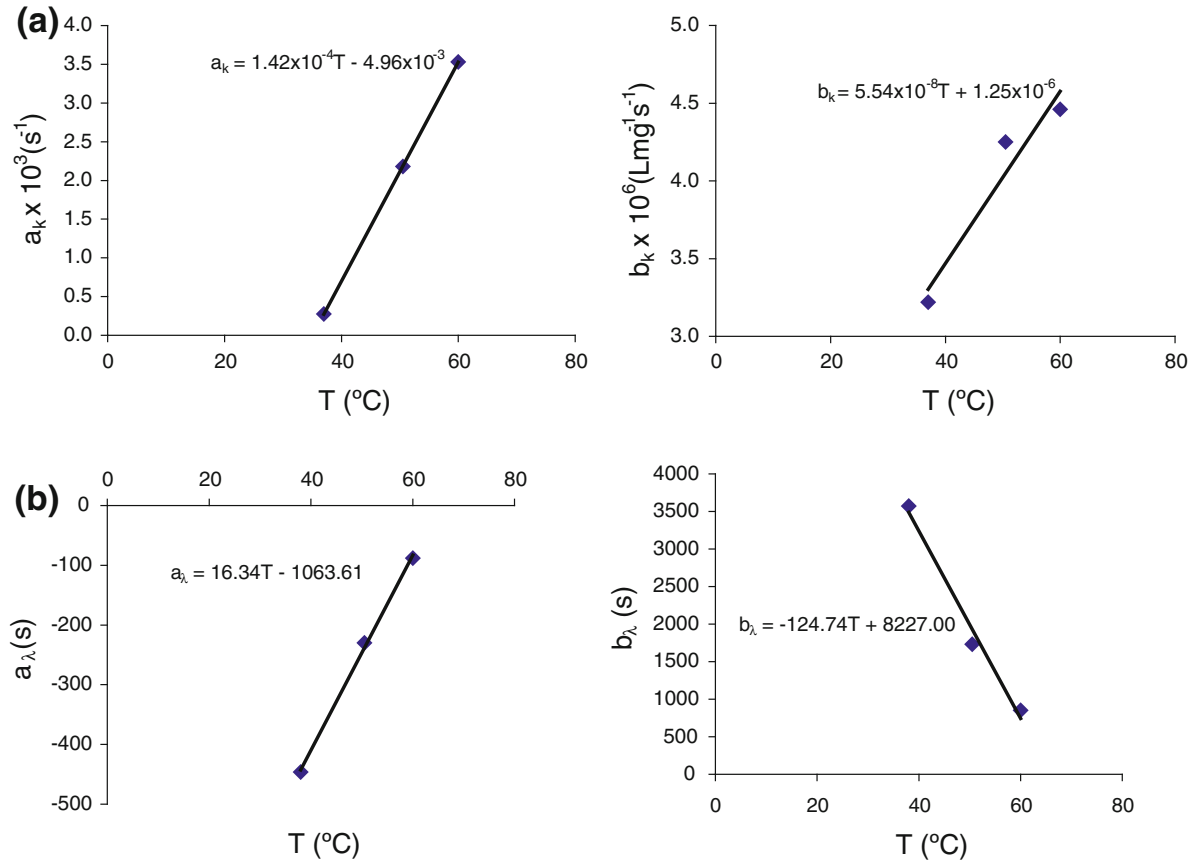

Fig. 5 Inactivation rate, $\mathrm{k}_{\max }$ (a) and shoulder, $\lambda$

(b) parameters estimated by the Gompertz model (Eq. 1) versus predicted values as a function of temperature and $\mathrm{EO}$ concentration (Eqs. 3 and 4, respectively)

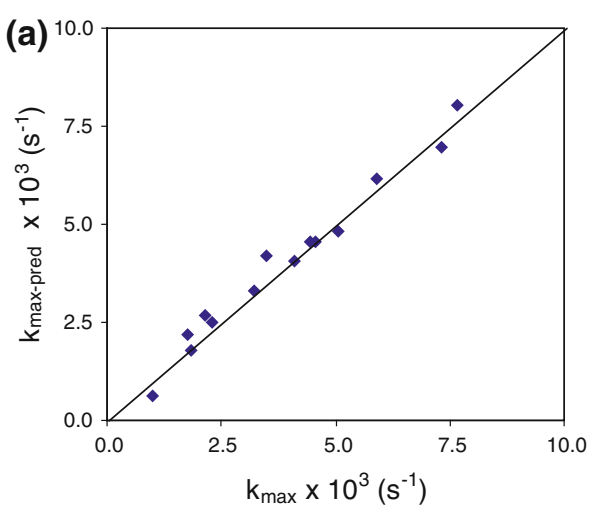

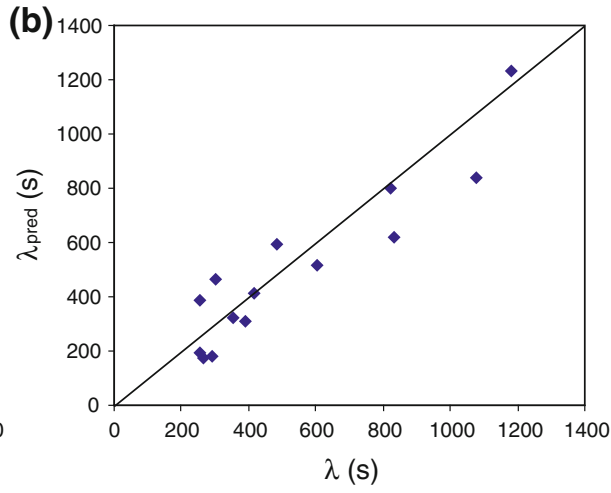

Or deducted for $\mathrm{U}$,

$\mathrm{U}=\lambda-\frac{(-7.5)}{\mathrm{k}_{\max } \times \mathrm{e}}\left\{1-\ln \left(-\ln \left(\frac{\mathrm{SAL}}{(-7.5)}\right)\right)\right\}$

where SAL is the sterility assurance level and [EO] corresponds to the EO concentration in the sterilizer headspace chamber. Once the diffusivity for this specific load is achieved, it would be possible to re-adjust this equation and replace this parameter by a general one that corresponds to the EO concentration where lethality of the process is being monitored. This effort would be more profitable if, simultaneously, equipment 
is developed to measure the EO concentration inside the load.

As already discussed, the tail parameter was assumed to be -7.5 .

The prediction of $B$. subtilis inactivation by the newly developed model can be visualized in Fig. 1 (for the seven first runs). The grey line was obtained considering the average values of temperature and EO concentration. One should be aware about difficulties in reproducing (and/or stabilizing) experimental conditions. Based on experimental results, it was found a variability of $4 \%$ for temperature and $14 \%$ for EO conditions. These allow considering that the temperature and EO concentration changes throughout all cycles are not significant and constant conditions may be assumed without compromise lethality prediction.

The predictive ability of the final model was assessed considering a band of prediction (upper and lower limits defined by considering the maximum fluctuations of temperature and EO concentration and calculated using Eq. 5).

These bands include the experimental data for all the conditions tested, which demonstrates that the inactivation of $B$. subtilis under EO sterilization can be successfully predicted using a model that only takes into account the process variables.

Although all efforts have been done in order to reproduce the experimental conditions of the different runs (similar industrial cycle design), the sterilizer does not operate as a Biological Indicator Evaluator Resistometer (BIER) vessel. Due to the dimension of this type of equipment (BIER), it generates reproducible results through reliable process control and monitoring, as well as a square wave EO sterilization cycle is attained, which would reduce the error related to the integrated lethality. In the sterilizer, the EO concentration corresponds to a condition obtained in its headspace and not to the one inside the load (as explained on Materials and methods- "Sterilization process"). The accuracy associated to the equipment used for monitoring the temperature and EO concentration also contributes to the overall predictive uncertainty.

\section{Conclusions}

The B. subtilis EO inactivation did not follow a first-order kinetics (i.e., linear inactivation). Experimental data showed an initial shoulder and a maximum inactivation rate period, and a Gompertz model was successfully applied in data fitting. The most relevant process variables that significantly affected the kinetic parameters were the temperature and the EO concentration. Mathematical models that describe the dependence of shoulder and maximum inactivation rate on such variables were also developed. These expressions were merged into the Gompertz model and accurate predictions of the original inactivation data were attained. Overall, an inactivation model expressed only in terms of the relevant process variables was achieved. The conventional design of EO sterilization cycles usually involves a significant amount of experimental work, which is time-consuming as well as expensive. The results of this work are certainly a contribution for an efficient control, design, and optimization of the EO sterilization process.

Despite the inexistence of standard guidelines on this topic, the predicted times for a target lethality should not be confined calculation of D-values, since non-linear tendencies occur.

Acknowledgments This study was supported by Bastos Viegas, S.A. The author Teresa R. S. Brandão acknowledges financial support from Fundação para a Ciência e a Tecnologia (SFRH/BPD/41419/ 2007).

\section{References}

1. Baranyi J, Roberts TA (1994) A dynamic approach to predicting bacterial growth in food. Int J Food Microbiol 23:277-294

2. Bhaduri S, Smith PW, Palumbo SA, Turner-Jones CO, Smith JL, Marmer BS, Buchanan RL, Zaika LL, Williams AC (1991) Thermal destruction of Listeria monocytogenes in liver sausage slurry. Food Microbiol 8:75-78

3. Box GEP, Hunter WG, Hunter JS (1978) Statistics for experiments: an introduction to design, data analysis and model building. Wiley, New York

4. Buzrul S, Alpas H (2004) Modeling the synergistic effect of high pressure and heat on inactivation kinetics of Listeria innocua: a preliminary study. FEMS Microbiol Lett 238:29-36

5. Chen H (2006) Use of linear, Weibull, and log-logistic functions to model pressure inactivation of seven foodborne pathogens in milk. Food Microbiol 24(3):197-204

6. Chhabra AT, Carter WH, Linton RH, Cousin MA (1999) A predictive model to determine the effects of $\mathrm{pH}$, milkfat, and temperature on thermal inactivation of Listeria monocytogenes. J Food Prot 62:1143-1149

7. Cole MB, Davies KW, Munro G, Holyoak CD, Kilsby DC (1993) A vitalistic model to describe the thermal inactivation of Listeria monocytogenes. J Ind Microbiol Biotechnol 12(3-5):232-239

8. Demitrius CA, Duran AP, Chamberlain VC, Hitchinc VM (1993) Comparison of European and US biological indicators for ethylene oxide sterilization. J Ind Microbiol Biotechnol 12:399-402

9. Garthright WE (1991) Refinements in the prediction of microbial growth curves. Food Microbiol 8:239-248

10. Geeraerd AH, Herremans CH, Van Impe JF (2000) Structural model requirements to describe microbial inactivation during a mild heat treatment. Int J Food Microbiol 59(3):185-209

11. Gibson AM, Bratchell N, Roberts TA (1987) The effect of sodium chloride and temperature on the rate and extent of growth of Clostridium botulinum type A in pasteurized pork slurry. J Appl Bacteriol 62(6):479-490

12. Gil MM, Brandão TRS, Silva CLM (2006) A modified Gompertz model to predict microbial inactivation under time-varying temperature conditions. J Food Eng 76(1):89-94 
13. Heider D, Gomann J, Junghann BU, Kaiser U (2002) Kill kinetics study of Bacillus subtilis spores in ethylene oxide sterilisation processes. Zentr Steril 10(3):158-167

14. ISO technical committee 198 (2006) ISO 11138-2, sterilization of health care products-biological indicators, part 2: biological indicators for ethylene oxide sterilization processes. ISO Press, Geneva, Switzerland

15. ISO technical committee 198 (2007) ISO 11135-1, sterilization of health care products-ethylene oxide, part 1: requirements for development, validation and routine control of a sterilization process for medical devices. ISO Press, Geneva, Switzerland

16. Juneja VK, Eblen BS, Marks HM (2001) Modelling non-linear survival curves to calculate thermal inactivation of Salmonella in poultry of different fat levels. Int J Food Microbiol 70:37-51

17. Juneja VK, Marks HM, Mohr T (2003) Predictive thermal inactivation model for effects of temperature, sodium lactate, $\mathrm{NaCl}$, and sodium pyrophosphate on Salmonella serotypes in ground beef. Appl Environ Microbiol 69(9):5138-5156

18. Kim SR, Rhee MS, Kim BC, Lee H, Kim KH (2007) Modelling of the inactivation of Samonella typhimurium by supercritical carbon dioxide in physiological saline and phosphate-buffered saline. J Microbiol Methods 70(1):132-141

19. Linton RH, Carter WH, Pierson MD, Hackney CR (1995) Use of a modified Gompertz equation to model non-linear survival curves for Listeria monocytogenes. Scott A. J Food Prot 58:946954

20. Mendes GC, Brandão TRS, Silva CLM (2007) Ethylene oxide sterilization of medical devices: a review. Am J Infect Control 35(9):574-581

21. Mosley GA (2002) Estimating the effects of EtO bier-vessel operating precision on D-value calculations. Med Devices Diagn Ind 24(4):46-56

22. Mosley GA, Gillis JR (2004) Factors affecting tailing in ethylene oxide sterilization part 1 : when tailing is an artifact ... and scientific deficiencies in ISO 11135 and EN 550. PDA J Pharm Sci Technol 58(2):81-95

23. Mosley GA, Gillis JR, Krushefski G (2005) Evaluating the formulae for integrated lethality in ethylene oxide sterilization using six different endospores forming strains of bacteria, and comparisons of integrated lethality for ethylene oxide and steam systems. PDA J Pharm Sci Technol 59(1):64-86

24. Mosley GA, Gillis JR, Whitbourne JE (2002) Calculating equivalent time for use in determining the lethality of EtO sterilization processes. Med Devices Diagn Ind 24(2):54-63

25. Mosley GA, Gillis JR, Whitbourne JE (2002) Formulae for calculations of integrated lethality for EtO sterilization processes. Pharm Technol 14(10):1-12

26. Mosley GA, Houghtling CW (2005) Interpreting and understanding microbial data in validation of ethylene oxide sterilization processes. Biomed Instrum Technol 39:466-482

27. Oxborrow GS, Placencia AM, Danielson JW (1983) Effects of temperature and relative humidity on biological indicators used for ethylene oxide sterilization. Appl Environ Microbiol 45(2): 546

28. Peleg M (2000) Microbial survival curves - the reality of flat "shoulders" and absolute thermal death times. Food Res Int 33:531-538

29. Peleg M, Cole MB (1998) Reinterpretation of microbial survival curves. Crit Rev Food Sci Nutr 38(5):353-380

30. Richardson T, Hyslop DB (1985) Enzymes. In: Fennema O (ed) Food chemistry. Marcel Dekker Inc, New York, pp 412-413

31. Rodriguez AC, Young B, Caulk K, Zelewski J, Kwasnica S, Aguirre S (2001) Calculating accumulated lethality and survivorship in EtO sterilisation processes. Med Devices Diagn Ind 23(9):100-107

32. Rutala WA, Weber DJ (1999) Infection control: the role of disinfection and sterilization. J Hosp Infect 43(Suppl):S43-S55

33. Shintani H, Tahata T, Hatakeyama K, Takahashi M, Ishii K, Hayashi $\mathrm{H}$ (1995) Comparison of $\mathrm{D}_{10}$-value accuracy by the limited Spearman-Karber procedure (LSKP), the Stumbo-Murphy-Cochran procedure (SMCP), and the survival-curve method (EN). Biomed Instrum Technol 29:113-124

34. Sordellini PJ, Satter SE, Caputo VA (1998) EtO sterilization: principles of process design. Med Devices Diagn Ind 20(12):47 